\title{
Marco comprensivo para el estudio de los procesos de formación de terapeutas
}

\author{
Diana M. Rodríguez Ch.* \\ J ulio Abel Niño Rojas \\ Universidad Santo Tomás
}

Recibido: noviembre 2 de 2005 Revisado: diciembre 14 de 2005 Aceptado: enero 23 de 2006

\section{Resumen}

La indagación sobre la formación contempla las intencionalidades del proyecto educativo considerado como contexto de formación, las modalidades con las que éste opera y sus presupuestos básicos, lo que constituye el modelo educativo y el impacto del mismo tanto en los terapeutas como en sus consultantes. Los procesos de formación y terapia se estudian como procesos de modelización compleja concebidos como el métodoproyecto para construir conocimiento, que articula los sistemas académicos y terapéuticos bajo la noción de isomorfismo en el escenario pedagógico de supervisión. Corresponde a una investigación cualitativa que creó escenarios metaobservacionales y reflexivos, cuyas escenas preexistían en los contextos de supervisión y supervisión estructurados en la Maestría en Psicología Clínica y de Familia de la Facultad de Psicología de la Universidad Santo Tomás. Los resultados muestran un proceso formativo gradual, integrado en la modalidad de supervisión y supervisión conjunta. Entre formados se comparten modelizaciones de la experiencia formativa y entre formadores circulan muy diversas concepciones de las modalidades formativas. Su eficacia gana en impacto a lo largo del tiempo, de lo que dan cuenta los indicadores del proyecto educativo.

* Correspondencia: Diana M. Rodríguez Ch., Docente Maestría en Psicología Clínica y de Familia, Facultad de Psicología, Universidad Santo Tomás, Bogotá, Colombia. Dianarodriguez@correo.usta. edu.co. J ulio Abel Niño Rojas, Docente Maestría en Psicología Clínica y de Familia, Facultad de Psicología, Universidad Santo Tomás, Bogotá, Colombia. julioabelnino@hotmail.com. 
Palabras clave: modelo sistémico, formación, terapeutas, proceso educativo.

\section{Abstract}

Investigation on training contemplates the intentionalities of the educative project, considered as training context, the operation modalities and basic assumptions that constitutes the educative model under which the therapists are trained and its impact both on therapists and on patients. Training and therapy processes are studied like complex modeling processes conceived as method-project to build knowledge that articulates the therapeutic and academic systems under the notion of isomorphism in the pedagogical setting of supervision. It corresponds to a qualitative research that created metaobservational and reflective settings whose scenes preexisted since it was a matter of the supervision contexts and joint supervision structured in Clinical and Family Psychology Master of the Psychology Faculty of Santo Tomás University. The results showed a gradual training process, focused in the modality of supervision that integrates, in a recent way, the joint supervision. Formative experience modelings are shared between students and among formers, circulate very diverse conceptions of formative modalities. Efficacy grows in impact through the time showed by educative project indicators.

Key words: systemic model, formation, therapist, educative process.

Tradicionalmente se ha concebido el proceso educativo y su efecto formativo como elemento fundamental para el desarrollo individual y colectivo, y se ha considerado que los aprendizaj es logrados por el educando y los niveles formativos que ellos le permiten escalar son indicadores adecuados de desarrollo tanto para el individuo como para un país. Es decir, se ha presumido la existencia de una relación lineal entre formación y desarrollo. Sin embargo las cifras del PNUD no respaldan esta idea (Ambrósio, 2003). Este problema ha constituido una de las críticas y búsquedas para científicos sociales. ¿Qué requiere ser comprendido y actuado para que la educación logre su propósito formativo, facilitador de desarrollo y bienestar colectivo e individual? Como lo plantea Maturana (1998), preguntarse «¿Qué queremos con la educación?», «¿Sirve la educación?».

En el dominio de la formación se han adelantado propuestas que pretenden asegurar el aprendizaje y la efectiva operación de éste en el campo de aplicación requerido. Uno de estos es el campo de la "clínica de las comunicaciones" (denominación propuesta por J. Miermont, 1987), donde confluye el

"cuerpo social de operadores sociales que tienen la responsabilidad de identificar, detectar, con fines de prevención, de acción, de sanción o reparación, el malestar de un individuo, familia o comunidad. En este campo los diversos modelos se fusionan. Ello ocurre cuando el estudio de los síntomas es atravesado por varios modelos pragmáticos: psicoanálisis, intervención sistémica, semiótica de la conducta, etoantropología comparativa" (p. 2).

Tal confluencia es convocada cuando se comprende que la familia y sus integrantes se enfrentan a regulaciones mutuas de orden biológico, cultural y social, todas ellas complejas de aprehender. 
Existe en el contexto internacional (American Psychological Association, 2002), como en el regional (Martínez - Mercado, 2002), la preocupación por conocer los modos en que se forman los terapeutas y la efectividad de sus intervenciones. La responsabilidad social del psicólogo clínico lo obliga a develar su articulación con el saber disciplinado ante la evidencia creciente de problemas de salud en la población. Esto es particularmente cierto para Colombia. El Informe de salud publicado en 1999 por la OPS, así como los estudios sobre salud mental de la Secretaria de Salud del Distrito (2005), señalan que el problema número uno del país en materia de salud se debe a causas externas, principalmente la violencia que afecta a toda la sociedad y que, la incidencia, severidad, grupos etarios e incapacidad por dificultades de este orden se incrementan y cronifican entre toda la población.

\section{Retos contemporáneos para la educación / formación}

La educación y la formación enfrentan la necesidad de dar cuenta de sus impactos al tiempo que están siendo interpeladas por los cambios vividos en los últimos años, que han transformado las estructuras de la vida económica, social y política. En este marco ha dominado una perspectiva económica de la educación, encaminada a considerar las exigencias de cualificación de los recursos humanos en un mundo globalizado y competitivo (Ambrósio, 2003).

Si bien se han constituido propuestas para integrar lo humano de una manera renovada en los requerimientos de orden político y económico, como las Teorías del Capital Humano, del Capital Social, del Desarrollo de Recursos Humanos, de la Educación para el Desarrollo, éstas aún se mantienen en el enfoque económico de la educación. Se confrontan con ellas las teorías más emancipadoras, donde la persona es el suj eto de su propia educación, que cuestionan la comprensión de la educación como vía y proceso de reproducción social y con ello los paradigmas de la transmisión de conocimientos y los modelos que separan formas y disciplinas del conocimiento, ignorando a los aprendices (Clenet, 2003).

Las nuevas políticas de educación necesitan introducir una comprensión de lo humano, donde hombres y muj eres sean comprendidos de manera integral, entendiendo entonces que la educación ha de preparar para ejercer una "ciudadanía activa". (Ambrósio, 2003). De acuerdo a Le Moigne (2000) se trata de reconocer que como ciudadanos somos capaces de producir conocimiento en y para la acción, que reflexionamos, que nos es posible modelizar de manera creativa, inteligible y a menudo juiciosa, lo inefable o lo indecible.

\section{La propuesta sistémica constructivista}

En el siglo XX se configuró la tradición constructivista con diversas corrientes, que coincidieron en centrar el énfasis sobre "el aprendiz", al entender que éste es "el actor de su propio saber" (Giordan, 2003). Desde la propuesta de Teresa Ambrósio (2003), la educación / formación en tal perspectiva requiere ser comprendida como una realidad personal que responda al reto de la inteligibilidad y de la intervención compleja, multidisciplinaria y multi-rreferencial. Para ello integra nuevos elementos: la comprensión del entorno, considerado eco-sistema complejo; de los fenómenos percibidos y representados, a través de procesos de modelización y modelos; y del proceso mismo de aprendizaj e que "construye coordinaciones intra - individuales" (Giordan, 2003). El espíritu del proyecto educativo de la Maestría se sitúa en esta tradición y por ello su vocación de estudiar y reconocer de manera continúa los modelos con los que se opera en los escenarios formativos y terapéuticos. 


\section{Complejidad y modelización}

El proceso formativo se plantea como uno complejo, tanto como el de aprendizaj e. Tal como lo afirma Clenet (2003) "intervenir en el dominio de la formación, en unión de los hombres, de la acción y de sus contextos, nos invita a reflexionar sobre una nueva forma de entendimiento: Ia inteligencia de la complejidad" (p. 2).

La complejidad se muestra como terreno fecundo para dar cuenta de estos procesos, pues la conjunción, la interdisciplina, la conectividad aparecen como principios claves para modelizar la formación. "Se trata de practicar el pensamiento complejo con la ayuda de la interdisciplinariedad, del conocimiento de las interdependencias sistémicas emergentes, practicar la reflexividad y la búsqueda de la intencionalidad y del sentido" (Ambrósio, 2003, p. 5). Este argumento expone bien los operadores para dar cuenta de una formación compleja.

Tanto formar como aprender implica un conj unto de funciones múltiples, complejas, polidistribuidas, policontextualizadas (Giordan, 2003). Esta manera de pensar cuestiona de entrada la legitimidad de los modelos tradicionales. Valga decir que un modelo se comprende en el sentido que lo exponen Miermont J . (1987) y Le Moigne (2002), es decir, es un proceso de modelización llevado a la práctica como facilitador para la imitación de un plan, de un esquema, permitiendo aprehender y reproducir eventos. Los modelos han sufrido diversos malentendidos: se les ha tomado por reales (confusión mapa - territorio); se han identificado con sus autores, como ocurre en la terapia, resultando en el disfraz de actitudes que caen fuera de contexto; se les ha tomado como referencia para las confirmaciones clínicas para confrontar ideologías opuestas; y han servido al eclecticismo y la incoherencia metodológica (Miermont, 1987).

Por ello el pensamiento complejo indaga por la pertinencia y coherencia de los modelos, buscando la legitimidad socio cultural de los conocimientos producidos (la investigación científica), transmitidos o reproducidos (la educación / formación) y la pues- ta en obra en las actividades humanas (las praxis).

Así como los modelos han enfrentado malentendidos, la modelización requiere para evitarlo dar cuenta de su epistemología. Es posible identificar a la hora actual dos comprensiones. Una enraizada en el pensamiento naturalista, positivista, y realista, que presume la existencia de los hechos que son dados a la consciencia, donde el modelo es la representación de los hechos, que a su vez lo legitiman independientemente del observador; se trata de una concepción ontológica del modelo (platónica) y bajo esta comprensión se privilegian los modelos formales lógico-matemáticos. Otra es la comprensión compleja de la modelización; ésta parte del argumento de que los "hechos, por ser hechos, debieron haber sido hechos, son por lo tanto construcciones que siguen las definiciones e interpretaciones y es la propia modelización la que legitima al modelo"; se trata de la concepción fenomenológica. Se le comprende como una modelización pragmática o heurística (Le Moigne, 2002). De ésta última nos ocupamos aquí.

En la educación/ formación compleja, estas nuevas formas de entendimiento no pueden más que expresarse a través de la modelización sistémica como una alternativa de "pret a former". Se trata entonces de aprender y de ayudar a aprender, a concebir y a construir "modelos-artefactos", hechos de símbolos a través de los cuales se trata de comprender inteligiblemente las diversas situaciones en las que se interviene. Los símbolos son artificios que pueden ser entendidos como "formas (sintaxis) que a la vez informan (semántica) y transforman (pragmática)" (Le Moigne, 2002, p. 3).

De esta manera se haría posible el círculo virtuoso entre formación y complejidad a través de la modelización: entender la formación en su complejidad y formar en la complejidad, sirviéndose de modelos "estructurantes - organizadores configuradores" (traducción de N. Emboussi del concepto de patrón propuesto por N. Hanson (1958 y 1977) y retomada por H. A. Simon (2001), citados por J. L. Le Moigne, 2003). 


\section{Eco-sistemas y modelización}

Tradicionalmente las ciencias sociales han considerado que el estudio de los procesos humanos requiere integrar el estudio del medio ambiente 0 del entorno. Desde la perspectiva analítica, el entorno fue dividido en partes (por ejemplo los estímulos, las situaciones), llegando a plantear una neta diferencia entre éste y el individuo. El pensamiento sistémico complejo busca recomponer éste fenómeno en su integridad, al reconocer las múltiples interacciones, retroacciones y emergencias. El interés es estudiar los sistemas humanos y las sociedades humanas. Para ello "la modelización sistémica propone el concepto de eco-sistema (posterior al de eco-organización), para dar cuenta de manera inteligible y practicable de lo que ha venido siendo designado como medio ambiente, para asociar el medio con el mundo del entorno (Le Moigne, 2004, p. 1).

Como lo propuso G. Bateson (citado por Miermont, 1987) una unidad de sobrevivencia evolutiva sólo puede ser considerada en la relación que el organismo mantiene con su entorno. Así se parte de concebir la integración de un individuo o grupo en su eco-sistema, es decir, su contexto de vida, material y humano. De esta manera, el entorno es una realidad organizadora, es el eco-sistema que es posible definir como "el conjunto de interacciones en el seno de una unidad geofísica, constituyendo una unidad complej a de carácter organizador o sistémica, que se organiza a partir de interacciones entre sus constituyentes" (Le Moigne, 2004, p. 2). Esto conlleva entender a las personas situadas en su contexto de vida también como modelizadoras de su eco-sistema, en un continuo proceso de indagación-acción, elaborando posibles elecciones.

En el marco del propósito de la presente investigación este eco-sistema se estudia a partir de: los actores entendidos como equipos de trabajo en escenarios de formación e intervención (de docencia y de supervisión) que despliega en sus acciones competencias propias (formación y terapia) y que implica el dar cuenta de los impactos que estas acciones generan (aprendizaj e y cambio).

\section{Aprendizaje y modelización}

Interrogarse sobre la formación, sea en el plano individual o colectivo, es interrogarse sobre el aprendizaje. Aquí se insertan condiciones de orden cognitivo: la construcción deliberada del próximo paso que revela la inteligencia humana, al lograr representarse a sí mismo en la acción. Tal capacidad simbolizadora de la auto-representación, permite la comprensión teleológica del mismo. Nuevamente aquí las capacidades cognitivas de representación se sirven de símbolos memorizables y computables que permiten identificar acciones posibles y que se construyen en los procesos de aprendizaje y cambio.

Situados sobre la persona, se evidencia que tal proceso es de naturaleza social: es un proceso interpersonal que es interiorizado y genera coordinaciones intra-individuales, es decir, reestructura el pensamiento del individuo. El pensamiento de una persona es pues eminentemente social, si bien se encuentra genéticamente programado no se desarrolla por razones puramente biológicas. Para el equipo de la Universidad de Génova liderado por André Giordan (2003), pensar complejamente el aprendizaj e se caracteriza por propiedades emergentes que se revelan en el estudio de las interacciones entre las condiciones del cerebro humano, la historia de pensamiento del aprendiz y las posibilidades ofrecidas por las situaciones educativas o culturales. A partir de ello propone comprender el aprendizaje en el intercambio de tres funciones: intencionalidad, elaboración y metacognición La intencionalidad, similar a la construcción deliberada del próximo paso (propuesta expuesta arriba por Le Moigne, 2004), se entiende como proyecto a su vez motivado por una necesidad, un deseo. Se sitúa aquí el afecto como motor del conj unto que dará peso a la acción y a la información. La elaboración, referida a los recorridos cognitivos que ocurren en la confrontación de la información que genera nuevos significados, opera por cambios discontinuos hasta la "comprensión de un nuevo modelo donde la estructura mental ha sufrido una metamorfosis. El marco de referencia de los cuestionamientos se ha progresiva- 
mente reformulado y la matriz de referencias reelaborado" (p. 6).

Esta nueva experiencia cognitiva se almacena y va movilizar y ser movilizada continuamente, reorganizando la memoria. El aprendiz dará nuevas significaciones al saber elaborado en la función de la metacognición, y así saberes y proyectos se situarán conjuntamente. De esta suerte, aprender es integrar información novedosa en una estructura de pensamiento ya existente y es a partir de ella que el aprendiz puede recabar, filtrar, decodificar, confrontar la nueva información. Se trata de un proceso de organización y regulación. Por supuesto todo ello requiere del entorno didáctico como contexto de aprendizaje.

\section{Cambio y modelización}

El cambio, como el aprendizaje, es entendido como un proceso discontinuo que implica la emergencia de novedad a partir de la interacción de los actores de un contexto, opera por saltos, lo que no se vincula solamente a programas o pasos establecidos de antemano (Boscolo y Bertrando, 1996). En esta perspectiva es posible plantear entonces que el tiempo se comprenda como teleológico, más que entrópico (la flecha del tiempo de Boltzman, donde el pasado condiciona el presente al impedir el retorno), o antrópico (jalonado por el futuro, la causa última). El tiempo se entiende como teleológico, construido en el presente. El tiempo es creación del proyecto de acción, que construye sentido para el accionar en curso (Le Moigne, 2004).

Por ello el cambio se encuentra en el dominio de lo fenomenológico, pues es una experiencia singular en cada individuo. Es entendido también en sentido batesoniano, como una noticia sobre una diferencia, tal como se entiende el conocer o la información en la cibernética.

En otros órdenes, en una comunidad que comparte puntuaciones sobre cómo y cuándo puntuar el cambio, este será susceptible de ser observado por otro, distinto a quien lo experimenta. De esta suerte el cambio puede ser modelizado al puntuar sobre las relaciones entre personas y en el comportamiento de los individuos, en un contexto relacional que construye significados y dota de sentido a la acción (Boscolo y Bertrando, 1996). Ello es particularmente importante en el contexto terapéutico que se ocupa de la promoción del bienestar; lo que en la gran mayoría de solicitudes de ayuda está atravesado por el alivio de síntomas, que si bien se vinculan con diversas interpretaciones de la realidad, también se encarnan en comportamientos y pautas reiteradas de relaciones. La construcción de nuevos sentidos/ acciones se hace entonces inteligible en los relatos de los actores (la experiencia vivida) y los comportamientos y relaciones estructuradas.

Es posible entonces distinguir dos niveles de cambio: en el orden del individuo y en el orden de las relaciones, y sin embargo, el uno no será posible sin el otro. El cambio social es un cambio en la configuración de acciones coordinadas que define la identidad del sistema en particular. En palabras de Maturana (en Elkaim, 1998) "el cambio social no tiene lugar sino cuando el comportamiento de los sistemas vivos individuales que lo componen se transforma, dando nacimiento a una nueva configuración de acciones coordinadas definidora de una nueva identidad para el sistema social" (p. 123).

\section{Modelización compleja de la educación/formación}

Reconocemos tres elementos claves en el proceso formativo: el ecosistema (que implica actores y contextos particulares de su accionar, agrupados en equipos y desplegando competencias), los procesos de modelización y sus impactos, entendidos en los escenarios formativos como el aprendizaje y co-aprendizaj e, y en los escenarios terapéuticos como cambio, vinculados los tres por procesos de modelización.

La formación implica una formación compleja del hecho mismo que ella es una realidad personal. Es decir se construye de manera intencional con frecuencia, aunque no siempre, en los distintos con- 
textos de vida y entornos, integra las experiencias de vida propia y ajena y ocurre a lo largo de toda la vida. Por ello es multicultural, interdisciplinaria, policontextual, multi-referencial, interdependiente tanto en el plano colectivo (social), como en el psicológico (intra individual).

Como proceso, se reconoce que ocurre en un tiempo de acción - creación situado, en un contexto de sentido (el entorno didáctico en situaciones deliberadas), y que las informaciones que allí circulan serán significadas y vinculadas con los contextos de vida del aprendiz. Como sistema vivo, el aprendiz y el que enseña, y las relaciones entre los dos, convocan tanto sus condiciones de orden biológico, como los procesos de orden cognitivo, cargados de emocionalidad y afecto, que discurren a través de la construcción de marcos de referencia y modelos (modelizaciones) continuamente en movimiento.

Si situamos estos procesos en el campo de la Clínica de la comunicación, propuesto por Miermont (1987), se hace necesario aportar pesos diferenciales a estos propósitos, en virtud de la responsabilidad social, al ser elemento constitutivo del cuerpo social de cuidado. Se hace necesario afinar los procesos de aprendizaje que tienen como escenario a las personas -ciudadanos-investigadores- interventores-, considerando que estos son animados y conducidos en escenarios colectivos (supervisión y seminarios); afinar los procesos evaluativos como estrategias de acompañamiento y fortalecimiento del aprendizaje que logren dar cuenta del plano individual y colectivo, tanto de manera cerrada como abierta, es decir como acciones finales y en proyecto; reconocer los entornos didácticos explícitamente, hacerlos inteligibles, comunicables y evaluables, así como los modos particulares de operar el aprendizaje de la complejidad. Es decir los modelos y modelizaciones que construimos para enseñar/ evaluar/ actuar.

\section{Intencionalidad y modalidad formativas: el isomorfismo entre los procesos académico y terapéutico}

La formación se ha planteado actualmente como el trabajo sobre procesos que articulan las diversas formas de aprender (aprender a aprender), de saber (ser y conocer) y de capacidades sociales (cooperar, dialogar, profundizar la democracia) (Ambrósio, 2003). Este acuerdo sobre las intenciones de la educación no se ha dado en relación con las modalidades de formación y, como lo afirma Le Moigne, (2003), se han evaluado sin considerar condiciones contextuales, como si se dijese "Proveamos la buena sintaxis, la buena semántica y seguirá de manera unívoca la buena pragmática" (p. 1).

En la formación de terapeutas se viene estudiando una modalidad ampliamente practicada: la supervisión. La supervisión directa implica que el supervisor guíe el proceso terapéutico en el mismo momento en el cual se desarrolla. Se constituye entonces, como lo afirma Everett, en un aspecto crítico de la maduración del campo de la terapia de la pareja y de la familia, al ofrecer un "ambiente para la socialización de la identidad y el aprendizaje de las habilidades", haciendo que los supervisores lleguen a ser los "cuidadores de la profesión". La formación bajo supervisión integra la formación técnica, científica, clínica y profesional, para formar una persona en capacidad de integrar la práctica empírica y clínica, y la evaluación de su acción en la medida en que interviene.

Como Io plantea Liddle (1988), es un proceso complejo que integra el estilo individual del aprendiz -estilos cognitivos-, la claridad conceptual de la formación -isomorfismo entre formación y terapia-, la prospectiva y evaluación sobre estudiantes y suprasistemas, y una mayor conciencia de las interconexiones entre varios ámbitos de la terapia familiar-especificaciones de los procesos de mutua influencia entre teoría, investigación, práctica y formación. 
Para la formadora Peggy Papp (citada por Elkaim, 1988), en el aprendizaje y la formación la supervisión es un proceso que enfrenta lo impredecible, comunica conocimiento, dirección, guía y al tiempo cuida de no privar al estudiante de su individualidad, creatividad e iniciativa. La complejidad de este proceso la conduj o a trabajar en grupos de consultores constituidos por pares, lo que denomina como un modelo de "colaboración en equipo», que es a la vez modelo de formación y tratamiento. Son los estudiantes quienes se convierten en equipo de consulta, en grupo de pares al contribuir a formular hipótesis y planificar intervenciones, siendo ellos los que tienen la última palabra sobre los casos. Se constituyen en grupo de reflexión. El supervisor trata de mantener al grupo en un contexto de pensamiento sistémico e integra las diferentes perspectivas. La responsabilidad final es del estudiante a cargo y del supervisor. Este tipo de supervisón le otorga más poder a los estudiantes, les enseña a colaborar entre colegas y a desarrollar una perspectiva sistémica. En el nivel de la formación, enseña a actuar y pensar de manera sistémica, trata de relacionar los diferentes problemas de la familia, lo que les inculca disciplina al hacer entrar las transacciones de la familia en el contexto de esa idea, lo cual los hace pensar en términos de las consecuencias de un cambio sistémico. Vive la experiencia de estar en plano de igualdad con su supervisor y esto permite reducir la ansiedad y disponer de gusto, cuestiones importantes en el proceso de aprendizaje.

J ay Haley (citado por Elkaim, 1988) resalta las conexiones entre los métodos de formación y las teorías terapéuticas enseñadas, a lo cual se refiere con el concepto de "naturaleza isomórfica". La noción de isomorfismo es importante pues permite considerar tales conexiones e interdependencias. Carlos Sluzki (1974) fue quien hizo una de las primeras afirmaciones acerca de la utilidad del concepto del isomorfismo, considerando que facilita el proceso de pensamiento que conduce al pasaje da una realidad interpersonal tanto en los programas planteados para modificar la epistemología del estudiante, como en los programas didácticos en terapia familiar.
Para Liddle (1988), el isomorfismo implica tres referentes. Primero, el paralelismo de los procesos referido a las descripciones a nivel procesual de las interacciones entre subsistemas. No se atribuye alguna acción o intencionalidad a las observaciones. Aquí los procesos paralelos son fenómenos significativos solamente en el sentido de las percepciones o valoraciones. El uso del término isomorfismo, por lo tanto, puede incluir la noción de que estas interacciones pueden ser modificadas: están suj etas a la intervención y al cambio intencional. Usando el isomorfismo como organizador cognitivo, el supervisor transforma las valoraciones de los procesos paralel os en una intervención, dirigiendo el comportamiento del terapeuta de modo que influencie las interacciones en varios niveles sistémicos.

En segundo lugar, la naturaleza isomórfica de la terapia y la formación es un principio que existe no sólo en el ámbito de las observaciones y las descripciones, también en el ámbito de la intervención en supervisión. El isomorfismo no se refiere simplemente a modelos que existen en el ámbito de la pura evaluación: Ias interacciones que se replican son implícitamente informaciones que pueden ser re-elaboradas. Son realidades que pueden ser modificadas cuando evaluación e intervención se hacen confusas. A conciencia y con intencionalidad el supervisor usa las informaciones derivadas de las interacciones (por ej emplo el modo en que las pautas problemáticas se replican en los contextos académicos y terapéuticos) para redireccionar el curso co-creado del sistema académico - terapéutico.

\section{Investigación}

Los posgrados en psicología y familia de la Facultad de Psicología de la Universidad Santo Tomás constituyen un contexto educativo donde se implementan una serie de estrategias pedagógicas para la formación de terapeutas en procesos de investigación/ intervención en contextos clínicos y de la salud mental, con la participación de 
diferentes sistemas humanos. Esta línea de investigación institucional, busca dar cuenta del modelo pedagógico de formación con el cual se entrenan terapeutas en el enfoque sistémico ecológico y contextual que caracteriza a la Maestría en Psicología Clínica y de Familia. Su intención es la de investigar los escenarios y contextos creados en la Maestría para dar cuenta de la formación de terapeutas sistémicos desde un enfoque sistémico complejo y el impacto de estos sistemas académico terapéuticos.

Los dominios de investigación son: la modelización, el co-aprendizaje y el cambio. De igual manera, los focos que permiten hacer las conexiones de los procesos son: la construcción de equipos de trabajo, el desarrollo de las competencias investigativas e interventivas y los impactos sociales en los diferentes actores y contextos. Los fenómenos de estudio están circunscritos a las consideraciones del contexto educativo de la Maestría, a las relaciones enseñanza/ aprendizaje, educador/ educando, supervisor/supervisados, terapeuta/familia, y a la diversidad factores situados en el orden contextual, interaccional e individual que caracterizarían las relaciones baj o estudio. En particular en el proyecto de modelos de supervisión, el objetivo es comprender los modos como se realiza el entrenamiento de los clínicos terapeutas.

\section{Preguntas de investigación}

- ¿Cómo las competencias de investigación/ intervención modelizadas en la supervisión construyen órdenes de cambio en los actores participantes?

- ¿Cómo los equipos generados en los contextos de supervisión posibilitan contextos de co-aprendizaje a través de las diferentes modelizaciones?

- ¿Cómo se construyen diversidad de impactos sociales a través de los procesos de cambio generados en los contextos de co-aprendizaje de supervisión?

\section{Método}

Se trata de un método metacontextual-reflexivo que privilegia los procesos metaobservacionales, los procesos conversacionales y la reflexión con los actores participantes en la formación de magísteres y en la psicoterapia.

\section{Participantes}

La población está conformada por los equipos de supervisores y los estudiantes matriculados en los diferentes semestres del programa de la Maestría y las familias atendidas por los estudiantes en los consultorios universitarios de la Facultad en atención ambulatoria.

\section{Instrumentos de observación y registro}

Formatos de evaluación de impacto: instrumentos para registrar las voces de los representantes de las instituciones y de las familias

Audio - video grabaciones: como un instrumento de apoyo al proceso anterior de registro de procesos conversacionales.

Protocolos: se trata de construcciones realizadas por el equipo investigador en conexión con los procesos reflexivos que se generan en los diversos escenarios.

Formatos de registros de observación: consisten en matrices de registro preparadas para recopilar la información que se produce en los diversos escenarios pedagógicos/ interventivos y generar con dicha información los procesos reflexivos con los equipos.

\section{Resultados}

Nos situamos en diversos niveles de observación: ante el propio proyecto educativo como eco-sistema para la formación y la terapia, ante los modos de operar los procesos de formación y de terapia 
baj o la modalidad de supervisión y supervisión conjunta (trabajo en co-equipos de supervisión), y ante los actores: supervisados y supervisores, y los procesos de modelización en la formación.

\section{Proyecto educativo: eco-sistema}

El proyecto es coherente a lo largo y a través del proceso de formación. Esto se observa en las intencionalidades de los diversos escenarios pedagógicos que recurren a los mismos principios operadores para el diseño de las escenas didácticas. Los textos del programa, así como los textos de los programas académicos, como los protocolos construidos para y en la supervisión y supervisión conjunta, se hallan impregnados de los principios, tanto como guía para la conceptualización, como para la acción y por ende para la comprensión/ transformación de los fenómenos objeto de estudio/ intervención.

Se devela en su accionar tanto en la escena formativa como interventiva el recurrir a equipos reflexivos, procesos conversacionales, autorreferencia. Las intencionalidades se sitúan en el plano de la acción y operan entonces como modalidades.

La inter-conectividad de todos los escenarios de formación de los terapeutas en la Maestría permite hacer un chequeo constante de los efectos de los diversos contextos de formación, con lo cual es posible reconocer en cada uno de los procesos de entrenamiento el logro de los objetivos y el cumplimiento de las competencias terapéuticas de los estudiantes.

\section{Procesos de formación y terapia: supervisión y supervisión conjunta}

Si bien se considera una interdependencia fundamental entre el sistema académico y el interventivo en la modalidad de supervisión, no se presenta, discute o reflexiona de manera explícita sobre ello en el equipo de supervisores. Esta lectura no está presente en los programas de formación, ni en las evaluaciones sobre tales procesos. Parecería que la supervisión toca más a la formación del terapeuta, como sistema de formación, haciendo destacar la figura del supervisor y la del supervisado más que el caso bajo atención. La modalidad de supervisión conjunta, por su parte, avanza en reflexiones sobre su encargo formativo - terapéutico y se destaca en ella el caso y las lecturas tej idas en relación con la solicitud de ayuda y la solución del problema.

\section{Los actores: supervisores y terapeutas en formación}

De manera particular para los supervisores, la supervisión es vivida como estrategia, recurso, modo de operar, actuar para la formación, donde se destaca como principio la contextualidad, la auto-referencia y el cuidado del estilo personal del terapeuta en formación. Para los estudiantes la supervisión es un escenario para la guía, el acompañamiento que en primer semestre cumple una función de «contención» y que luego avanza en momentos de «contención y libertad». Contexto formativo que facilita el auto-conocimiento y la solidaridad para el trabajo en equipo, que se centra en el desarrollo de habilidades y recursos para el conocimiento/ comprensión de las demandas de ayuda y la construcción de soluciones para los problemas de los consultantes. Un contexto que requiere por una parte de un supervisor claro, cercano, que guíe, que no tema retroalimentar y, por otra, supervisados dispuestos a la experiencia de aprender. Para los estudiantes en primer semestre la figura central del proceso es el supervisor y para el segundo año de formación este lugar será ocupado ya por el equipo de supervisión, como red de colaboración.

En relación con la supervisión conjunta los estudiantes la caracterizan como una manera de acompañar, modelar en la diversidad, de dinamizar el aprendizaje, las dinámicas de los equipos y las personas, como un sistema de terapeutas puestos en acción de aprender, co-equipos trabaj ando gracias a la diversidad. Todo ello es visto como generador de novedad. En este contexto no se percibe tan protagónicamente la figura del supervisor, los 
protagonistas son los equipos y el caso. Para los supervisores, la supervisión conjunta facilita la modelización, construye el conocimiento en la acción, genera conocimiento a partir de las diferencias y dilemas y facilita construir el pensamiento sistémico en los supervisados, quienes trabajan en integrar lo diverso para la acción, por ello restituye la solidaridad entre pensar y actuar. Facilita el trabajo en equipos reflexivos y la metaobservación Para ambos, supervisados y supervisores, es claro el impacto sobre los casos bajo esta modalidad pues recompone las escenas terapéuticas.

En la modalidad de supervisión conjunta, los supervisores recurren para su diseño a juego de roles, videocintas de casos, simulaciones y construcción de mapas. Su principal recurso es la complementariedad, pues el trabajo de dos equipos invita a la multicontextualidad y la interdisciplina, lo que enriquece la construcción de hipótesis en una perspectiva interaccional. En coherencia con ello los estudiantes destacan que su mayor aprendizaje radica en la conectividad, es decir, el desarrollo del pensamiento sistémico complej 0 .

\section{Indicadores de eficacia del proyecto educativo}

Los siguientes datos de la evaluación semestral son parte del estudio de impacto de la formación de terapeutas de la Maestría en Psicología Clínica y de Familia en los consultorios de psicología de la Universidad Santo Tomás, los cuales son sistematizados por el Coordinador de Prácticas de la Maestría, Ps. Orlando González.

El número de casos efectivamente atendidos incrementa de I a IV semestre en una proporción de $13.4 \%$ a $20.3 \%$ El promedio de sesiones por caso va de 3.9 en I a 5.6 en IV. El cierre de los casos por logro de obj etivos terapéuticos aumenta con el paso del tiempo de $3.1 \%$ en I semestre a $10.6 \%$ en IV semestre, pasando por un valor de $8.6 \%$ en II y III. El cierre del caso por abandono del consultante aumenta con el tiempo de $1.2 \%$ en I a 3.9\%en IV. EI número de sesiones realizadas de manera continua es similar en I y II semestre, una a tres sesiones, que pasa luego de una a cuatro en III semestre y de una a tres y a cinco en IV semestre. La continuidad en las sesiones termina para I semestre en la séptima, en II semestre en la octava, para III semestre en la número 12 y para IV semestre en la número trece. Entre 2004 y 2005 ha aumentado el número de casos efectivamente atendidos, ha disminuido la inasistencia y el retiro de los consultantes, se mantiene similar el abandono de los mismos. Se han aumentado las horas efectivas de entrenamiento realizadas en todos los semestres.

\section{Conclusiones}

El proyecto educativo de la Maestría en Psicología Clínica y de Familia evidencia, a través de sus redes conversacionales e instrumentos de evaluación (indicadores de logro), un mej oramiento continuo en la eficacia de su proceso formativo - terapéutico, en un contexto consistente y coherente que educa en el paradigma de la complejidad de manera longitudinal y transversal. Ello da cuenta del círculo virtuoso entre el proyecto, sus modelos de formación y sus modalidades. Es decir, entre el ecosistema, los principios-intencionalidades y las modalidades con las que opera.

El proceso formativo logra una aj ustada interdependencia para la eficacia entre formación y terapia a lo largo del tiempo. Es decir, el isomorfismo logra su cometido modelizador de los dos procesos conexos, haciendo que el estudiante - terapeuta en formación gane en eficacia a lo largo de la formación sobre dos años.

La modalidad que aparece como privilegiada, «la unión de todo», como lo describe un estudiante, es la supervisión. Ésta destaca la figura del supervisor en I semestre, donde se le percibe en un rol 
de contención y guía a través de la cercanía emocional, lo que se diluye en II semestre, para en III y IV semestre disponer en su lugar del equipo de supervisión como guía aliada, equipo terapéutico para la atención efectiva de los consultantes. En esta modalidad se percibe entonces la jerarquía del supervisor y destacan las figuras clásicas de profesor y aprendiz, se preocupa por las coordinaciones intraindividuales y los procesos cognitivos del estudiante, particularmente en primer semestre. Se comprende como espacio formativo que trabaja en la deconstrucción y co-elaboración del paradigma de la complejidad para la comprensión de los problemas y sistemas humanos en su ecología.

Por su parte la supervisión conjunta, modalidad reciente en la Maestría, es percibida como un contexto innovador que enriquece el aprendizaje, ya que posibilita y trabaja en la diversidad y la conexión de manera multicontextual. Allí el estudiante se siente más tranquilo en un contexto de relaciones más heterárquicas. Destaca el estudio y atención de los casos, privilegiando más el proceso te- rapéutico, y conservando su cuidado en el proceso formativo, pues recurre a equipos reflexivos, de autorreferencia y metaobservación como estrategias pedagógicas - terapéuticas. El isomorfismo terapéutico se revela de manera más clara y se integra al diseño de las escenas.

Los indicadores del proyecto dan cuenta de una eficacia creciente, con espacios de mejoramiento en los procesos de recepción y sostenibilidad del proceso (abandono de los consultantes y sesiones no realizadas). La eficacia de los terapeutas claramente se incrementa a lo largo del proceso formativo en el que perciben que ganan en auto-conocimiento, conectividad y reflexividad, integrando el paradigma de la complejidad en su versión de lo humano.

El siguiente mapa permite apreciar gráficamente las comprensiones que desde la línea/ proyecto se han visualizado como fenómeno/ problema de investigación e intervención.

\section{Modelos contextuales de formación de terapeutas desde un enfoque sistémico}

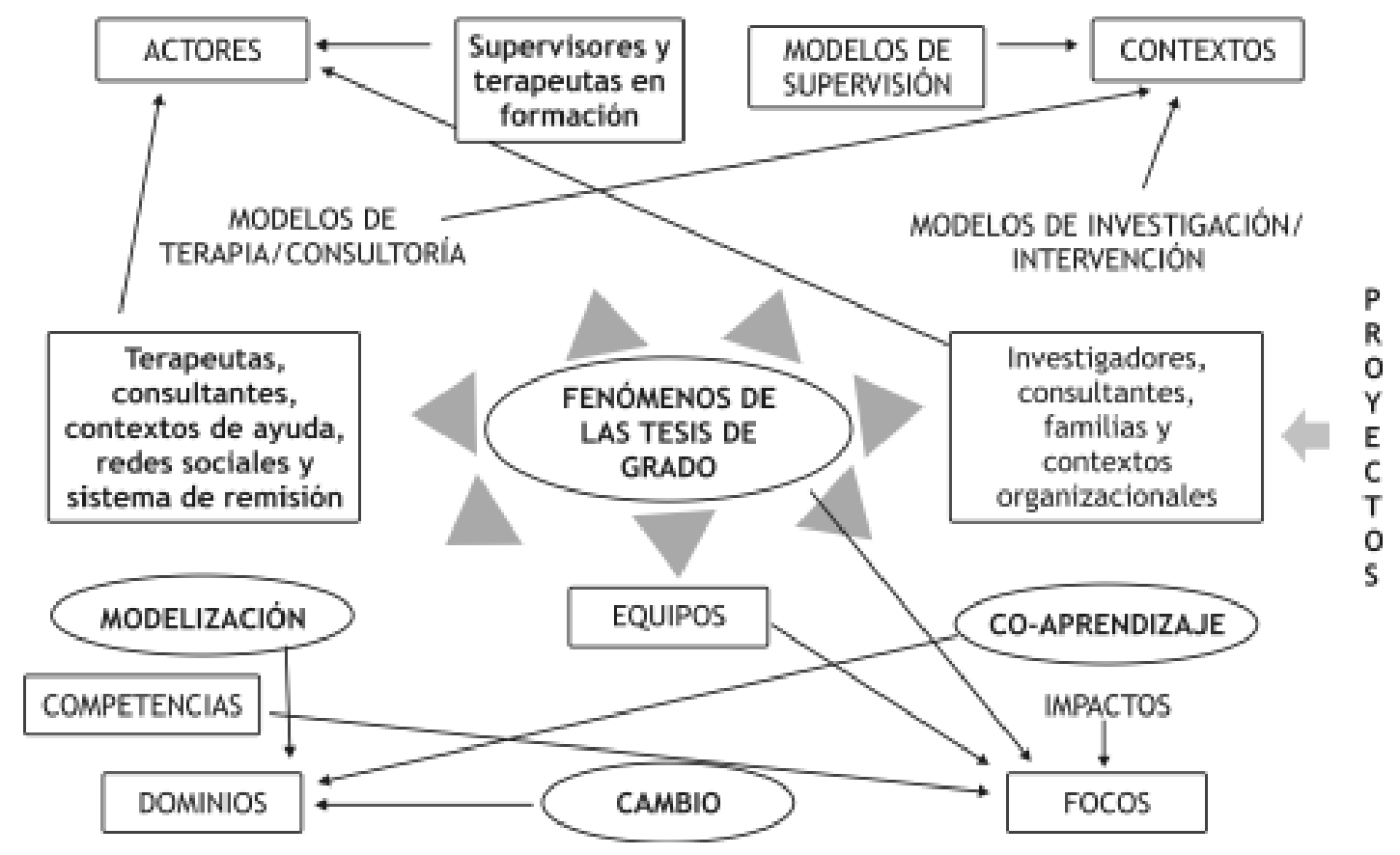




\section{Referencias}

American Psychological Association. Speciality Principles. APA (Online). (Recuperado 13 de agosto de 2002). http:// www.apa.org/ crsppp/specprinciples. html

Ambrósio, T. (2003) Sur la complexité des relations entre la formation de la personne et le développement durable de la société. Conference au Grand Atelier MCX "La formation au défi de la complexite". Lille, France.

Boscolo, L. y Bertrando, P. (1996) Los tiempos del tiempo. Barcelona: Paidós.

Clenet, J. (2003) La formation au défi de la complexité. Interroger et modéliser les intervenctions de formation en situations complexes. France: Editorial du Reseau Intelligence de la Complexité.

Colombia: basic country health profiles, Summaries 1999. Pan American Health Organization (2001). http:// www. paho. org/ English/ SHA/ prflcol.html (2002, junio 24).

Elkaim, M. (1988) Formaciones y prácticas en terapia familiar. Buenos Aires: Nueva Visión.

Elkaim, M. (1998) La terapia familiar en transformación. Barcelona: Paidós.

Giordan, A. (2003) Complexité et apprendre, formations professionelles et entreprises apprenants". LDES Université de Geneve. Conference au Grand Atelier MCX La formation au défi de la complexite". Lille, France, 18-19 de septiembre de 2003.

Gurman, A. y Kniskern, D. (1995) Manuale di terapia della famiglia. Italia: Boringhieri. Formazione e supervisione in terapia della famiglia (Howard A. Liddle)
Le Moigne, J. L. (2000) Sur I'entendement de la complexité: restaurer la modelisation dans nos cultures et pratiques. Editorial La lettre chemin faisant. MCX., $N^{\circ} 37$, J uillet.

Le Moigne, J. L. (2003) Sur I'intelligence de la complexité en formation. Contribution au Grand Atelier MCX, "La formation au défi de la complexite". Lille, France, 18-19 de septiembre de 2003.

Le Moigne, J. L. (2002) L'epistemologie de la modelisation. France: MCX.

Le Moigne, J. L. (2004) Les trois temps de la modelisation des éco-systemes: I'entropie, I'antropique et le téléologique. France: MCX. Aix Provence Cedex.

Maturana, H. (1998) Emociones y lenguaje en educación y política. Santiago de Chile: Dolmen.

Mercado Martínez, F. J . (2002) Investigación cualitativa en America Latina: Perspectivas críticas en salud. http:// ualberta.ca/ ijqm/ (2002, febrero 14).

Miermont, J . (1987) Quelques questions posées aux modéles.

Politica Distrital de Salud Mental (2003) http:// www. saludcapital.gov.co (2003, julio 13).

Plan de investigación institucional de la familia (PLANFA) (2001) Universidad Santo Tomás, Facultad de Psicología. Posgrados en Psicología clínica y de familia.

Línea/ proyecto institucional de investigación: Modelos contextuales de la formación de terapeutas desde un enfoque sistémico y ecológico, 2005. USTA. 